\title{
Three-Dimensional Architecture of Lithium-anodes made from Graphite Fibers Coated with Thin-films of Silicon Oxycarbide: Design, Performance and Manufacturability
}

Ibrahim Saleh ${ }^{\mathrm{a}}$ and Rishi Raj ${ }^{\mathrm{a}}$

${ }^{a}$ Department of Mechanical Engineering

University of Colorado at Boulder

Boulder, CO 80309-0427, US

\section{Revision to Journal of Power Sources}

December 2015

\section{Abstract}

Silicon oxycarbide $(\mathrm{SiCO})$ is an amorphous molecular network of Si-C-O tetrahedra anchored to graphene-like carbon. The graphene forms a three dimensional cellular network with a domain size of $\sim 5 \mathrm{~nm}$. Therefore nanometer thick films of $\mathrm{SiCO}$ grown on graphite may be expected to have unusual behavior. We grow these films on a bed of commercially available graphite fibers that serve the dual function of a current collector. The electrochemical behavior of the composite is measured as a function of the thickness of the SiCO films. Thick films approach the typical behavior of bulk SiCO (which has three times the capacity of graphite, but suffers from poor first cycle efficiency). However, films, approximately $100 \mathrm{~nm}$ thick, show high first cycle efficiency as well as high capacity. The composite performs better than the prediction from the rule-of-mixtures, which further substantiates the unusual behavior of the thin-film architecture. The Raman spectra of these thin films also differ from bulk SiCO. The development of thin graphite fibers, with a high surface to volume ratio that have the same capacity as the current graphite-powder technology, coupled with manufacturing of these thin-films by a liquid-polymer precursor based process, can propel these results toward commercialization.

Key words: Lithium-ion batteries, anodes, silicon oxycarbide, thin films, graphite and graphite fibers. Corresponding author: rishi.raj@colorado.edu; (303)492-1029. 


\section{Introduction}

The silicon-oxycarbides $(\mathrm{SiCO})$ are a relatively new anode material for $\mathrm{Li}$-ion batteries. They have the following special attributes,

(i) Their capacity ranges from 500 to $1000 \mathrm{mAh} \mathrm{g}^{-1}$, which varies with the molar concentrations of the constituents [1], with the highest values being obtained near the middle of the C-Si-O composition triangle - since they are amorphous and their composition varies, their capacity cannot be prescribed a theoretical value.

(ii) They have a pseudo-amorphous, molecular network structure constructed from a cellular graphene-like carbon, with a domain size of $\sim 5 \mathrm{~nm}$. Tetrahedral units of silicon, carbon and oxygen are anchored to the graphene [2]. Thus at the molecular level they comprise a combination of silicon, carbon and $\mathrm{SiO}_{\mathrm{x}}$, which, on their own, are candidates for anode materials. An analysis of Li-capacity data spanning a wide range of compositions, coupled with a nanodomain model for the structure of SiCO compounds, has shown that their high capacity arises from the migration of $\mathrm{Li}$ to the mixed-bond $\mathrm{C}-\mathrm{Si}-\mathrm{O}$ tetrahedral units [3].

(iii) SiCO materials have low density, typically $2 \mathrm{~g} \mathrm{~cm}^{-3}$, and, therefore, an open molecular network, which enhances the diffusivity of Li within it [4], leading to good performance at high C-rates [5]. The low modulus and low volumetric expansion upon intercalation, which has been measured to be less than $8 \%$ (unpublished), endows very good cyclic stability and high resistance to failure. The chemical inertness of SiCO promotes safety.

(iv) The silicon oxycarbides are made from silicone resins, also known as siloxanes and silsesquioxanes. The silicon-carbon-oxygen chemistry is built into these liquid polymers and can be varied widely [6]. They convert to the inorganic phase when heated to $800-1000{ }^{\circ} \mathrm{C}$. Thus they are potentially low cost, not only as raw materials, but also from the point of view of manufacturability. The liquid polymer route can open the way for fabricating nanocomposites of various kinds - for example by mixing the precursor with particles of silicon - to further improve performance.

Up till now SiCO has been implemented as anodes in powder form. The polymer precursor is cross-linked by heating to $\sim 300{ }^{\circ} \mathrm{C}$, milled into powders and then pyrolyzed by heating in an inert atmosphere at $\sim 800{ }^{\circ} \mathrm{C}$, which are then processed into anodes in the same 
way as graphite powders. The SiCO powders show high capacity with three times the capacity of graphite, good C-rate performance, and cyclic endurance, but suffer from low first cycle efficiency (65\%-75\%), and large hysteresis between charge and discharge cycles $(0.75 \mathrm{~V}-1.25 \mathrm{~V})[7]$. Since the intrinsic structure of these materials consists of graphene networks on the scale of $\sim 5 \mathrm{~nm}$, we wondered if films of them grown on graphite may lead to better properties. (Earlier work on thin films deposited on copper substrates did not show an improvement [8].) At the same time a novel processing approach also presented itself where we were able to deposit thin films of SiCO using a flash-pyrolysis method where a small amount of the liquid precursor was sprayed onto a web of carbon fibers and pyrolyzed in just a few seconds at $800{ }^{\circ} \mathrm{C}$ [9]. The nearly perfect wetting of all surfaces of the fibers within the fiber-matt allowed all fibers to be immediately covered with thin films of SiCO. Films of nanometer thicknesses could be made by diluting the polymer precursor in a solvent and reducing the amount of this solution sprayed on to graphite-fiber matt. Successive cycles of deposition, repeated in quick succession, could be used to build up the film thickness to the desired level.

The choice of graphite fibers in these experiments was limited by those available offthe-shelf. These widely available fibers are optimized for making graphite-reinforcedpolymer-composites for structural applications; they are far from being optimized for Li sequestration. In the experiments reported below, we have therefore characterized their electrochemical behavior, as a baseline for the evaluating the results after they have been coated with thin films of SiCO. The premise of these experiments was that the nature of the interface between the graphite and the SiCO films grown on it may influence the structure of the films. Thus the synergy between the fibers and the coatings are studied in two ways, (i) first by increasing the thickness of the coating, expecting that whereas thick coatings would tend toward the bulk behavior of SiCO, thin coatings may reflect the nature of the graphiteSiCO interface, and (ii) by adding the Li capacity from the fibers and from the coating using the rule-of-mixtures, to see if the thin-film behavior deviates from it.

\section{Preparation of the anodes}

The anodes were prepared with Toray TGP-H-030 graphite paper. According to the manufacturer the fiber mesh has only $20 \%$ fill density, with an overall physical density of 0.4 
$\mathrm{g} \mathrm{cm}^{-3}$. The fibers are impregnated with a polymer resin. The paper is specified to have a flexural strength of $40 \mathrm{MPa}$, and flexural modulus of $8 \mathrm{GPa}$. The average fiber diameter was $7 \mu \mathrm{m}$. The polymer residue in these fibers (5\% PTFE) was removed by heat-treating at 1000 ${ }^{\circ} \mathrm{C}$ in argon for one hour. Coupons, 0.5 " in diameter, were punched from the fiber-fabric. The fiber mesh was brittle and fragile after this heat treatment procedure. The coupons weighed $6.5-7.0 \mathrm{mg}$ apiece.

The polymer precursor for SiCO, 1 3,5,7-tetramethyl-tetravinylcyclotertrasiloxane (TTCS) was purchased from Gelest, USA. It was mixed with $1 \mathrm{wt} \%$ dicumyl peroxide, which served as a catalyst for cross-linking. If needed the precursor was diluted with THF, a common solvent.

The SiCO-GF anodes were prepared in the following way. A drop of the precursor solution was placed on the fiber coupon. It spreads immediately over all surfaces of the fibers in the mesh. It is then cross-linked at $\sim 170{ }^{\circ} \mathrm{C}$, and finally pyrolyzed at $\sim 800{ }^{\circ} \mathrm{C}$ when it converts into the amorphous SiCO ceramic phase. The last step was carried out in two ways, (i) by gradually heating the specimen, at a rate of $10^{\circ} \mathrm{C} \mathrm{min}^{-1}$ to the pyrolysis temperature, and holding for $5 \mathrm{~h}$ in argon atmosphere, and (ii) by flash pyrolysis where the specimen was exposed to approximately $800{ }^{\circ} \mathrm{C}$ under a radiant furnace for a few seconds. The latter process was conducted within a glove box in an argon atmosphere with oxygen content of less than 1 ppm. Both methods gave identical electrochemical behavior. Before the coating was applied the 0.5 " diameter coupons were fragile, but after the coating they were mechanically resilient and could be bent without fracture.

The thickness of the coating could be anticipated by the following analysis. We write the coating thickness as , the fiber- diameter as $d$, and the weight fraction of SiCO as $w_{S}$, the density of graphite and $\mathrm{SiCO}$ as ${ }_{G}$ and $\mathrm{SiCO},{ }_{S}$. Consider a single fiber of a unit length; its weight is given by $\left(d^{2} / 4\right){ }_{G}$. Since $\square d$, the weight of the coating is given by $d s$. Therefore,

$$
w_{S}=\frac{d{ }_{S}}{\left(d^{2} / 4\right)_{G}+d{ }_{S}}
$$

Rearranging Eq. (1) leads to the following result 


$$
\bar{d}=\frac{1}{4} \frac{G}{{ }_{S}} \frac{w_{S}}{1 w_{S}}
$$

The fiber diameter was approximately $7 \mu \mathrm{m}$. Therefore $w_{S}$ of $5 \%$, and $10 \%$, translates into

$=100 \mathrm{~nm}$ and $200 \mathrm{~nm}$, setting $\left({ }_{g} /{ }_{S i C O}\right) \quad 1$ since both have a density in the $2.0-2.3 \mathrm{~g}$ $\mathrm{cm}^{-3}$ range.

Micrographs of the graphite mesh before and after coating with $11 \mathrm{wt} \% \mathrm{SiCO}$, along with their Raman spectra are shown in Fig. 1. The enhanced contrast in Fig. 1(b), relative to bare fibers in 1(a), is most likely induced by the greater electron reflectivity of higher molecular weight SiCO. The Raman spectrum of the coated fibers is remarkably different from that of the bare fibers. The coating shows a sharp $\mathrm{G}$ peak, with broad shoulders that reach far, while the fibers show the characteristic D and G peaks of graphite. The Raman spectrum for $\mathrm{SiCO}[10]$ is included in 1(c); it is similar to that of the graphite fibers. In summary the dual $\mathrm{D}$ and $\mathrm{G}$ peaks seen in powder-SiCO, are replaced in the SiCO films by a single $\mathrm{G}$ peak with broad reaching shoulders on either side of the peak.

\section{Electrochemical testing}

The coupons prepared in the way described above are ready for electrochemical testing since the graphite fibers also serve as the current collector; the mesh makes electrical contact with the bottom cap in the CR2032-type coin cell. The liquid electrolyte permeates through the entire thickness of the electrode. Lithium-metal foil was used as the counter electrode. Soulbrain-1.0M LiPF in EC/DEC=1/1 (V/V) was the electrolyte, and WhatmanGlass Microfiber the membrane-separator. The batteries were assembled, crimped and closed in argon atmosphere.

The principal variable in the electrochemical characterization was the current density, which ranged from $15 \mathrm{~mA} \mathrm{~g}^{-1}$ to $\sim 600 \mathrm{~mA} \mathrm{~g}^{-1}$, corresponding to absolute values of $0.1,0.5$, 1, 2.5 and $5 \mathrm{~mA}$. (The current densities are expressed in terms of the total weight of the anode equal to the sum of the weight of the graphite fibers and SiCO.) The electrochemical cycles were constrained between $3 \mathrm{~V}$ and $0.01 \mathrm{~V}$. In one instance we also tested the absolute capacity by holding the cell at $0.01 \mathrm{~V}$ at the end of the current cycle until saturation was achieved. 
The specific capacity, first cycle efficiency, hysteresis and cyclic fading were measured. Baseline data were obtained for uncoated graphite-fiber coupons. The electrochemical properties of the composite were measured as a function of $w_{S}$, the weight fraction of SiCO. The data for the composites were analyzed by the rule-of-mixtures.

\section{Results}

In a typical experiment the cells were subjected to 10 cycles. (In one instance the cyclic durability was tested for GF-blank and GF-SiCO for more than 300 cycles.) The Coulombic efficiency and the capacity were measured as a function of the current density and the weight fraction of SiCO. The Coulombic efficiencies, after the first cycle, were always very close to $99.9 \%$, and are therefore not reported; they can be approximately judged from the cyclic data, which is included in the graphs. The first cycle efficiency, however, is an important measure of performance, and is reported in detail. The hysteresis between the charge and the discharge cycles was measured as the voltage difference at the mid-point of the final capacity.

\subsection{Capacity, First Cycle Efficiency, and Fading: a Comparison}

Results from GF-blank and GF-SiCO are compared in Fig. 2(a) - 2(d). In all instances the composite shows a significantly higher capacity than the GF-blank. The graphs in 2(a) and 2(b) compare the data obtained at $\sim 15 \mathrm{~mA} \mathrm{~g}^{-1}$, and at $\sim 400 \mathrm{~mA} \mathrm{~g}^{-1}$. Figure 2(c) shows the change in the capacity with a continuous increase in the current density. Figure 2(d) gives the cyclic durability at $81 \mathrm{~mA} \mathrm{~g}^{-1}$; note that while GF-blank fails after $\sim 275$ cycles, the composite continues to perform well at $>300$ cycles.

The variability in the capacity in Fig. 2(c) is notable. Lowest variability and the highest capacities are obtained at $16 \mathrm{~mA} \mathrm{~g}^{-1}$. The variability is somewhat abated by the SiCO coating, but remains an unusual feature of these results. We have conjectured that perhaps the electrical resistance of the fiber bed may have varied because of the uncertainty in the mechanical force that is used to press the coin cells. Therefore we measured the change in the electrical resistivity of the fiber bed under mechanical pressure. The specimens for these measurements were cut into $32 \mathrm{~mm}$ x $32 \mathrm{~mm}$ squares from the graphite paper. The thickness of the paper was $110 \mu \mathrm{m}$. Silver paint to the opposite edges of the sheet; the electrical 
resistance was measured between these edges. Pressure was applied by placing a weight on the paper. The voltage was measured as a function of the current up to $250 \mathrm{~mA}$; the overall slope was linear supporting Ohmic behavior. The resistivity of the uncoated fiber mesh was $0.036-0.040 \Omega \mathrm{cm}$ without the pressure, and $0.031-0.035 \Omega \mathrm{cm}$ with the pressure. The resistivity of the coated fiber mesh was $0.042 \Omega \mathrm{cm}$ and remained unchanged under pressure. Therefore, electrical resistance cannot explain the variability in the electrochemical performance. We now suspect that bubbles that may have become trapped within the fiber mesh during electrolyte infiltration, resulting in variable performance. Future experiments will continue to explore the origins of this variability.

The hysteresis of bulk $\mathrm{SiCO}$ and SiCO films deposited on copper show a high hysteresis, in the range of $0.75 \mathrm{~V}$ to $1.25 \mathrm{~V}[7,8]$. In Fig. 2(a) it is about $0.25 \mathrm{~V}$. We find such low hysteresis to be a consistent feature of the GF-SiCO electrodes.

\subsection{The Influence of SiCO wt. $\%$}

The capacity, the first cycle efficiency and the hysteresis voltage were measured as a function of $w_{S}$, at similar values of the current density, in the range $70-90 \mathrm{~mA} \mathrm{~g}^{-1}$. These plots are shown in Fig. 3(a), (b) and (c), respectively. The weight fraction of $\mathrm{SiCO}, w_{S}$, was varied from $4 \%$ to more than $50 \%$. Interestingly, the performance parameters remain essentially independent of the weight fraction for $4 \% w_{S} \quad 8 \%$; this implies that the performance is dominated by the properties of the graphite-SiCO interface rather than by the thickness of the film.

The first cycle efficiency of these thin-film GF-SiCO electrodes varies from $85 \%$ to $90 \%$; in comparison the values for bulk SiCO range from $65 \%$ to $75 \%$ [1,5]. Likewise the hysteresis for these thin films is only about $0.1 \mathrm{~V}$ whereas the values for bulk SiCO are much higher $(0.75-1.25 \mathrm{~V})$. These are very significant differences between bulk SiCO and thinfilm GF-SiCO.

The graphs is Fig. 3 refer to current densities of $70-90 \mathrm{~mA} \mathrm{~g}^{-1}$. The capacity and the shape of the electrochemical cycles at lower and higher current densities, for $w_{S}=8.5 \% \quad 10 \%$ are shown in Fig. 4. Most noteworthy here is that hysteresis remains low even at high currents (in bulk SiCO hysteresis becomes more severe with higher current). Data for 10 
cycles are presented here. The near ideal overlap of the cycle shapes shows that the Coulombic efficiency is close to $100 \%$.

\subsection{Capacity with voltage hold at $0.01 \mathrm{~V}$.}

The capacity of the GF-SiCO, $w_{S}=10 \%$, and GF-blank were measured after holding the cell at $0.01 \mathrm{~V}$ until the current dropped down to negligible values. The Li was intercalated into the electrodes at a current density of $90 \mathrm{~mA} \mathrm{~g}^{-1}$. The capacity of the GF-blank increased from $150 \mathrm{mAh} \mathrm{g}^{-1}$ to $175 \mathrm{mAh} \mathrm{g}^{-1}$, while that of GF-SiCO increased from $230 \mathrm{mAh} \mathrm{g}^{-1}$ to 285 $\mathrm{mAh} \mathrm{g}^{-1}$ when held at $0.01 \mathrm{~V}$.

\subsection{Thick film GF-SiCO - comparison with bulk (powder)}

Thick films are expected to approach the behavior of bulk SiCO, which is measured with powders. The results for powder-SiCO are compared with GF-SiCO $\left(w_{S}=48 \%\right)$ in Fig. 5 . At this weight fraction the capacity is dominated by $\mathrm{SiCO}$; therefore the capacity has been normalized with respect to the weight of the SiCO. Data for ten cycles are given. Two observations are of note. The cycles in the composite overlap nearly exactly, indicating nearly ideal coulombic efficiency; the performance of the powder anode shows some drift suggesting a slightly less than full coulombic efficiency. Second, the hysteresis in the composite is $\sim 0.6 \mathrm{~V}$ as compared to nearly $1 \mathrm{~V}$ in the powder-based anode. Given the high weight fraction of $\mathrm{SiCO}$, these differences could not have arisen from the graphite-SiCO interface. It is likely that they arise from the morphology of the SiCO phase in the composite, which, at this high weight fraction, was in the form of a web-like structure spread continuously over the conducting fibers.

\subsection{Surface area capacity in $\mathrm{mAh} \mathrm{cm}^{-2}$}

Since the electrolyte permeates throughout the graphite-fiber bed, and since all fibers serve as current collectors, the integrated capacity of the GF-SiCO anodes is expected to continue to increase as the thickness of the graphite-fiber bed is increased. The implication is that the capacity expressed in terms of the surface area of the electrode can be increased to very high values, essentially without limit. We have tested this expectation by measuring the capacity for 2, 3, 4, and 5 layer stacks of GF-SiCO anodes, each one individually being the same as used in the experiments described in the earlier figures. In this instance the capacity is expressed in terms of the surface area of the electrodes. The results are described in Fig. 6 
where the surface capacity is shown to increase linearly with the number of layers in the stack.

\subsection{Prediction from the Rule-of-Mixtures}

The rule-of-mixtures prediction is analyzed by combining the data from GF-blank and from bulk $\mathrm{SiCO}$, that is, by assuming that the capacities of the fibers and $\mathrm{SiCO}$ add as if they were independent of each other. According to the rule-of-mixtures we have that

$$
q^{*}(V)=\left(1 \quad w_{S}\right) q_{G F}(V)+w_{S} q_{S i C O}(V)
$$

Here $q^{*}(V)$ is the gravimetric capacity of the composite, measured as a function of the voltage. Similarly, $q_{G F}$ and $q_{S i C O}$ are the capacities of graphite-fiber and SiCO powder at the same voltage, measured independently, at the same current density. Equation (3) may now be applied to the entire electrochemical cycle.

The results from the rule-of-mixtures, calculated from Eq. (3), are given in Fig. 7. The capacities for GF-blank and for SiCO powder are shown in the Figs 7(a) and 7(b). The prediction from the rule-of-mixtures is compared with experiment in Fig. 7(c). The measured performance is much better than predicted by the rule-of-mixtures. The first cycle efficiency is higher, as is the reversible capacity. The reduction in the hysteresis is very significant.

\section{Discussion}

In this section we discuss the following topics: (i) the performance of the GF-SiCO thin-film architecture, ostensibly related to the nature of the graphite-SiCO interface, (ii) the design of the GF-SiCO architecture in order to enhance the gravimetric and volumetric capacities of the anodes, and (iii) manufacturability and scale-up.

\subsection{The GF-SiCO interface}

Bulk SiCO powders are endowed with high capacity, nearly three times that of graphite, high cyclic stability and the ability to perform at very high C-rates without failure $[1,5,7]$. However, they suffer from low first cycle efficiency (65 -75\%) and high hysteresis $(0.75-$ 1.25 volts). As shown in Fig. 3, thin-films of SiCO, represented by a weight fraction of less than $\sim 8 \%$, lead to first cycle efficiencies of $85-95 \%$ and hysteresis of less than $0.2 \mathrm{~V}$. Furthermore, with these thin-films the capacity becomes essentially independent of the 
thickness, implying that the behavior is dominated by the graphite-SiCO interface. According to Eq. (2) these films have a thickness of $100-200 \mathrm{~nm}$. These results have both scientific and technological implications.

The unusual nature of these thin films is underpinned by the Raman spectra shown in Fig. 1(c). Bulk SiCO powders show a pattern that is similar to that of the graphite fibers with dual $\mathrm{D}$ and $\mathrm{G}$ peaks that are distinctly separated. Instead the thin films show one, sharp G peak with broad shoulders that extend widely on either side of the peak. The pointed G peak resembles graphene. Graphene is a zero band-gap material; perhaps the electrical conductivity of these thin-films, which is necessary for electron transfer from the surface of the films to the graphite substrate, is explained by the presence of this G peak.

The key question of course is how the structure of the films can be influenced by the graphite substrate. Here two points can be made. The intrinsic structure of SiCO consists of a cellular network of graphene with a domain size of $\sim 5 \mathrm{~nm}$. This graphene network evolves when hydrogen is removed during pyrolysis leaving behind dangling bonds of carbon; then, carbon, being insoluble in silica, segregates and self-assembles into the graphene network. It is therefore quite conceivable that the presence of the graphite surface alters this evolution to create a different structure. Secondly, recent first principles calculations of the structure of SiCO has predicted a low energy 2D structure shown in Fig. 8 [11]. This structure has a very high density of mixed-bonds between C-Si-O, which are known to sequester large amounts of $\mathrm{Li}[2]$. It can be postulated that these 2D layers begin to form on the graphite surface, to some extent, and continue to form until the films gets quite thick.

\subsection{Design considerations: gravimetric and volumetric capacity}

The SiCO thin-film on graphite-fiber architecture of the anodes offers new design and materials selection opportunities to enhance their performance. The fibers in the present experiments, which have been developed for fiber reinforced polymer composites, have only one third the capacity of the state-of-the-art graphite-powders used in Li-ion batteries. It is well known that carbon made from different sources has widely different capacity for Li [12].

The selection of the precursors of SiCO is likely already optimized in view of the experiments on materials with a wide range of chemistries [1]. However, the relationship between the precursor chemistry and the growth of thin films on graphite substrates is not known. 
The most obvious benefits are likely to come from the geometrical design of the anode architecture, which has two main elements, the fiber diameter, $d$, and the packing density of fibers in the mesh, $f$. The film thickness should also be considered, but, in view of the results presented here, we shall assume that the films are thin so that the capacity is controlled essentially by the interface; this is rather a strong assumption and, therefore, the approximate nature of the analysis below must be kept in mind. The assumption simplifies the analysis considerably, since now the capacity is proportional to the surface area in the composite measured as a function of the weight, or the volume. The gravimetric surface area is written by the well recognized parameter, $S S A$, or the specific surface area, commonly expressed in units of $\mathrm{m}^{2} \mathrm{~g}^{-1}$. For fibers it can be immediately written that

$$
S S A=\frac{4}{d_{g}}
$$

Using the form of Eq. (3) we may then write that

$$
q^{*}=\frac{d^{2}}{4}{ }_{g} q_{G F}+\left(\frac{4}{d_{g} j}\right)_{S i C O}
$$

where ${ }_{S i C O}$ is the capacity of the film per unit area of the film, with units of $\mathrm{mAh} \mathrm{m}{ }^{-2}$. Note that the term is the brackets represents $S S A$. Inspection of Eq. (5) shows that smaller values of $d$ would make the second term larger than the first. If we assume that the second term is dominant then a two-fold reduction in fiber diameter would lead to a two fold increase in the capacity of the composite, and so on.

The volumetric capacity of the composite, $\quad$, expressed in $\mathrm{mAh} \mathrm{cm}^{-3}$ is given by a simple transformation of Eq. (5) by converting the weight into the volume

$$
{ }^{*}=f_{g} q^{*}
$$

where $f$ is the packing factor $\left(=0.2\right.$ in the present experiments). Note that in Eq. (6) $q^{*}$ is in units of $\mathrm{mAh} \mathrm{g}^{-1}$, and $g$ in units of $\mathrm{g} \mathrm{cm}^{-3}$. 
Finally, We compare the present results to the case of a standard electrode made from $10 \mu \mathrm{m}$ thick copper foil coated on both sides with $44 \mu \mathrm{m}$ thick graphite powder as the active material; in this case the area capacity (of the total anode) is estimated to be $3.5 \mathrm{~mA}$ $\mathrm{cm}^{-2}$, the gravimetric capacity as $150 \mathrm{mAh} \mathrm{g}^{-1}$, and the volumetric capacity as $350 \mathrm{mAh} \mathrm{cm}$. In the present anodes the gravimetric capacity of about $240 \mathrm{mAh} \mathrm{g}^{-1}$ exceeds this benchmark. However, the volumetric capacity of the present electrodes is a factor of three to four lower. The packing density of the current fiber paper was $20 \%$. It can be increased by a factor of three. Furthermore graphite fibers of a smaller diameter would increase the gravimetric capacity, which from Eq. (6), immediately translates into a higher volumetric capacity, as well.

\subsection{Manufacturabiity and scale-up}

The simplicity of one-step processing of the SiCO-on-fiber anode, which can be immediately inserted into the battery, is notable. The anode is mechanically resilient - it bends without fracture - and can be stored in air. The additive manufacturing approach, described in [9] is already being developed to deposit thin-films of controlled thicknesses on the nanoscale on graphite-fiber substrates. Two special features of this approach are (i) the liquid precursor solution spreads throughout the fiber mesh because of its wetting behavior, and (ii) the ultra thin films of the polymer can be pyrolyzed in just a few seconds without forming defects. The scale-up of this approach for coating large sheets of graphite paper is under consideration [9].

\section{Summary}

(i) Thin films of SiCO, which is a candidate material for anodes, are deposited as continuous layers on the surfaces of graphite fibers, where the fibers serve the dual function of the current collector. The liquid electrolyte permeates through the entire thickness of the fibermesh providing an intimate, large area interface for the electrochemical reaction. Unexpectedly, the films are apparently electrically conducting for the transfer of electrons from the surface of $\mathrm{SiCO}$ to the graphite substrate lying underneath.

(ii) Raman spectra show that the thin films have a different structure that bulk SiCO. They show a sharp G peak similar to graphene. The D peak, seen in bulk SiCO, is replaced by broad shoulders on either side of the G peak. First principles calculations suggest the 
possibility of two-dimensional structures where a layer of $\mathrm{Si}$ and $\mathrm{O}$ atoms covers a sheet of graphene [11], shown in Fig. 8. It is possible that the growth of $\mathrm{SiCO}$ on graphite from polymer precursors invokes structures of this kind. Films that range in thickness from $100-$ $200 \mathrm{~nm}$ yield capacities that are essentially independent of the film thickness (see Fig. 3), suggesting a strong role of the interface structure in electrochemical performance.

(iii) The thin-film architecture consistently shows much higher first cycle efficiency (85$90 \%$ ) and lower hysteresis $(0.1-0.2 \mathrm{~V})$, than bulk SiCO. The equivalent values for bulk SiCO are $65-75 \%$ and 0.75 to $1.5 \mathrm{~V}$.

(iv) The one-step manufacturing of the anodes can be cost-effective and conducive to scaleup [9]. The anodes are mechanically resilient and can be stored in air. Additive manufacturing of the composites is facilitated by the wetting behavior of the polymer precursor and "flash pyrolysis" for its conversion into SiCO.

(v) Equations (5) and (6) provide guidelines for increasing the gravimetric and volumetric capacity by reducing the fiber diameter and increasing the packing density in the fabric.

\section{Acknowledgements}

It is a pleasure to acknowledge the contributions form Dr. Lung-Hao Hu who was the first to demonstrate the possibilities of the three-dimensional architecture. We are grateful to the Ceramics Program in the Division of Materials Research at the National Science Foundation for supporting this research, in its entirety, under Grant No. DMR-0907108. 


\section{References}

1. A. M. Wilson, G. Zank, K. Eguchi, W. Xing, J. R. Dahn, Pyrolysed siliconcontaining polymers as high capacity anodes for lithium-ion batteries, Journal of Power Sources, 68 (1997) 195-200.

2. Saha, A., Raj, R., \& Williamson, D. L. (2006). A Model for the Nanodomains in Polymer- Derived SiCO. Journal of the American Ceramic Society, 89(7), 2188-2195.

3. P. E. Sanchez-Jimenez, R. Raj, Lithium Insertion in Polymer-Derived Silicon Oxycarbide Ceramics, Journal of the American Ceramic Society, 93 (2010) 1127-1135.

4. Weidman, P. D., Ahn, D., \& Raj, R. (2014). Diffusive relaxation of Li in particles of silicon oxycarbide measured by galvanostatic titrations. Journal of Power Sources, 249, 219-230.

5. Ahn, Dongjoon, and Rishi Raj. "Cyclic stability and C-rate performance of amorphous silicon and carbon based anodes for electrochemical storage of lithium." Journal of Power Sources 196, no. 4 (2011): 2179-2186.

6. Fukui, Hiroshi, Hisashi Ohsuka, Takakazu Hino, and Kiyoshi Kanamura. "Influence of polystyrene/phenyl substituents in precursors on microstructures of $\mathrm{Si}-\mathrm{O}-\mathrm{C}$ composite anodes for lithium-ion batteries." Journal of Power Sources 196, no. 1 (2011): 371-378.

7. D. Ahn, R. Raj, Thermodynamic measurements pertaining to the hysteretic intercalation of lithium in polymer-derived silicon oxycarbide, Journal of Power Sources, 195 (2010) 3900-3906.

8. Shen, J., and R. Raj, R. (2011). "Silicon-oxycarbide based thin film anodes for lithium ion batteries." Journal of Power Sources 196 (14), 5945-5950.

9. Zoli, L., Sciti, D., Liew, L. A., Terauds, K., Azarnoush, S., \& Raj, R. (2015). Additive Manufacturing of Ceramics Enabled by Flash Pyrolysis of Polymer Precursors with Nanoscale Layers. Journal of the American Ceramic Society, online DOI:10.1111/jace.13946. 
10. Cross, T., Raj, R., Prasad, S. V., \& Tallant, D. R. (2006). "Synthesis and tribological behavior of silicon oxycarbonitride thin films derived from poly (urea) methyl vinyl silazane." International journal of applied ceramic technology 3 (2), 113-126.

11. Yu, L. and Raj, R. (2015). On the Thermodynamically Stable Amorphous Phase of Polymer-Derived Silicon Oxycarbide, Scientific Reports, srep14550.

12. Dahn, J. R., Zheng, T., Liu, Y., \& Xue, J. S. (1995). Mechanisms for lithium insertion in carbonaceous materials. Science, 270(5236), 590. 


\section{Figure Captions}

Figure 1. (a) and (b) Micrographs of GF-blank and GF-SiCO (c) Raman spectra for the fiber mesh, the coated fiber mesh, and SiCO by itself.

Figure 2. A comparison of SiCO-GF performance relative to GF-blanks. Note that the fiber based anodes have a higher capacity (a), lower hysteresis (b), less variability (c) and much better resistance to failure (d).

Figure 3. The influence of SiCO wt. \% on (a) the capacity, (b) first cycle efficiency (b), and (c) hysteresis. Significant improvement over SiCO powder is seen for less than 12 wt. \%.

Figure 4. Influence of current density on the cyclic behavior of SiCO ( 10 wt. \%)-GF composite anode.

Figure 5. (a) The cyclic behavior of SiCO powder anode made by the conventional method. (b) The cyclic behavior of a composite with 48 wt.\% SiCO at the same current density. The capacity has been normalized with respect to the SiCO weight. At this composition the contribution of the graphite fibers to the capacity is minor.

Figure 6. Since all graphite fibers are current collectors, and since the electrolyte makes contact with all of them, the thickness of the anode can be increased without limit to increase the area capacity of the anodes. This thesis is affirmed in these data where the area capacity increases linearly with the number of layers in an anode stack.

Figure 7. (a) The capacity of GF-Blank and (b) SiCO powder tested separately. (c) The capacity of the composite compared with the calculation from the rule-of-mixtures that combines the results in (a) and (b), using Eq. (3).

Figure 8. A 2-D crystal of monolayers of silicon on graphene with a binding energy of $-1.2 \mathrm{eV}$ per silicon atom. The stoichiometry of this structure is $2: 4: 3:: \mathrm{Si}: \mathrm{C}: \mathrm{O}$ [11]. 


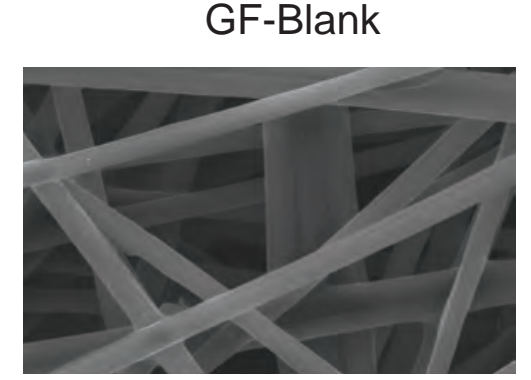

$20 \mu \mathrm{m}$

(a)

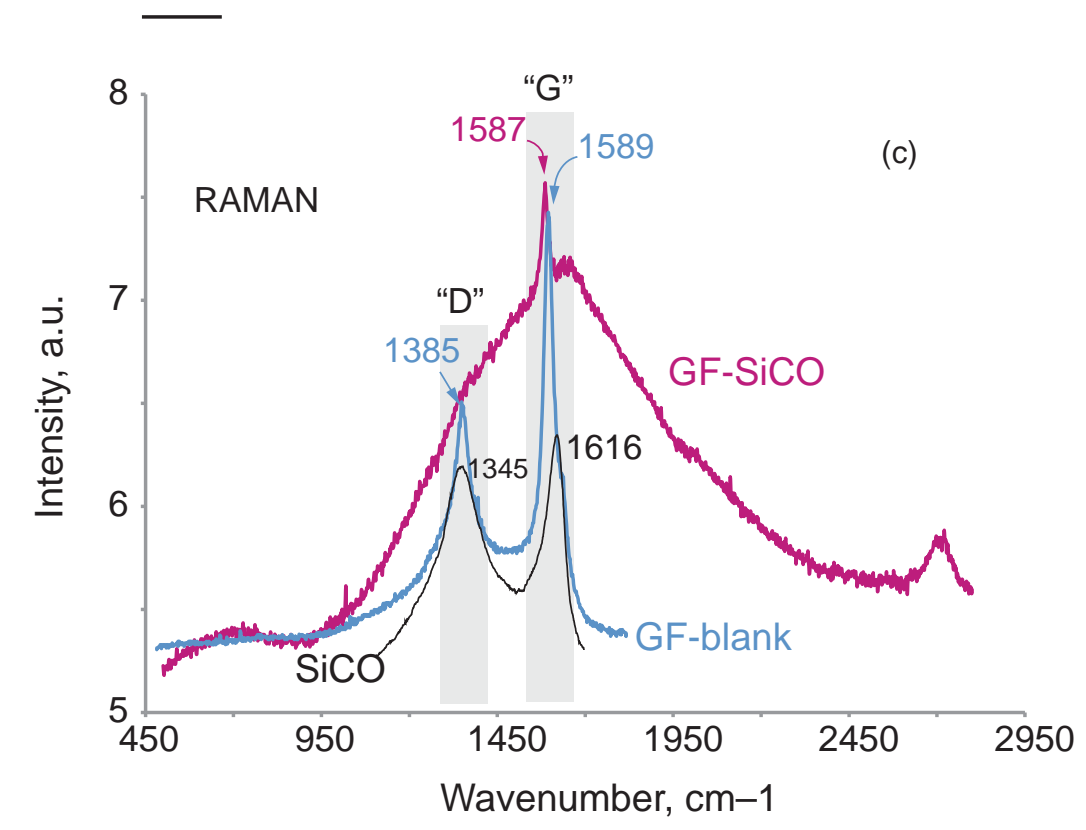

(b)

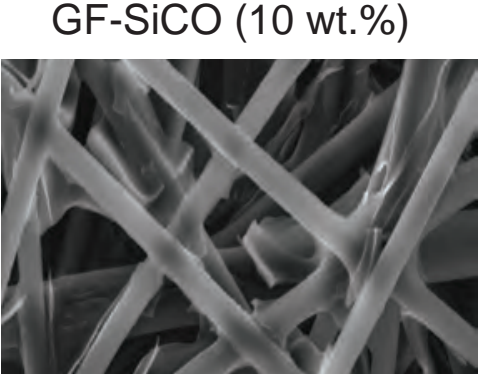

Figure 1. (a) and (b) Micrographs of GF-blank and GF-SiCO (c) Raman spectra for the fiber mesh, the coated fiber mesh, and SiCO by itself. 

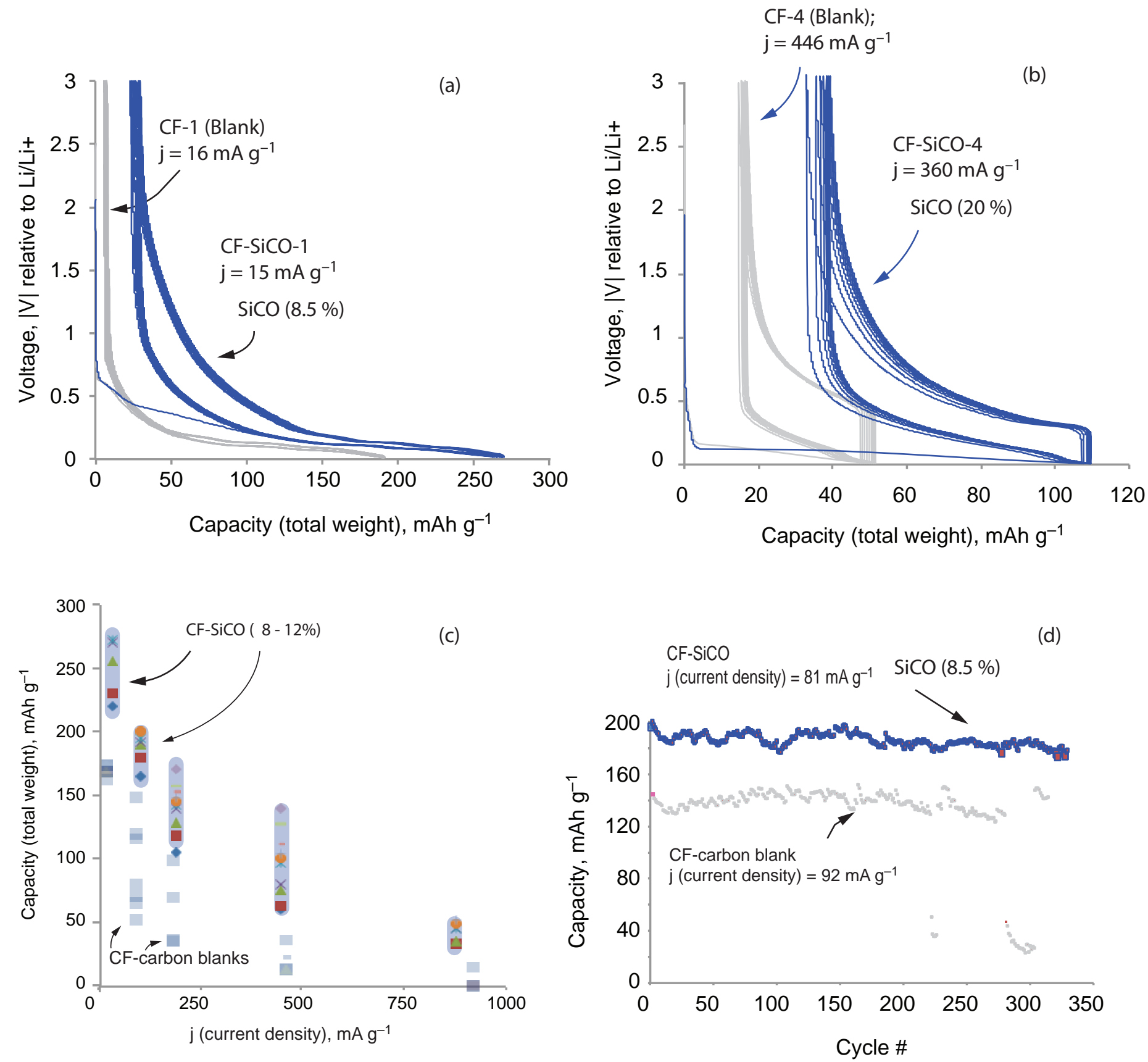

Figure 2. A comparison of SiCO-GF performance relative to GF-blanks. Note that the fiber based anodes have a higher capacity (a), lower hysteresis (b), less variability (c) and much better resistance to failure $(\mathrm{d})$. 

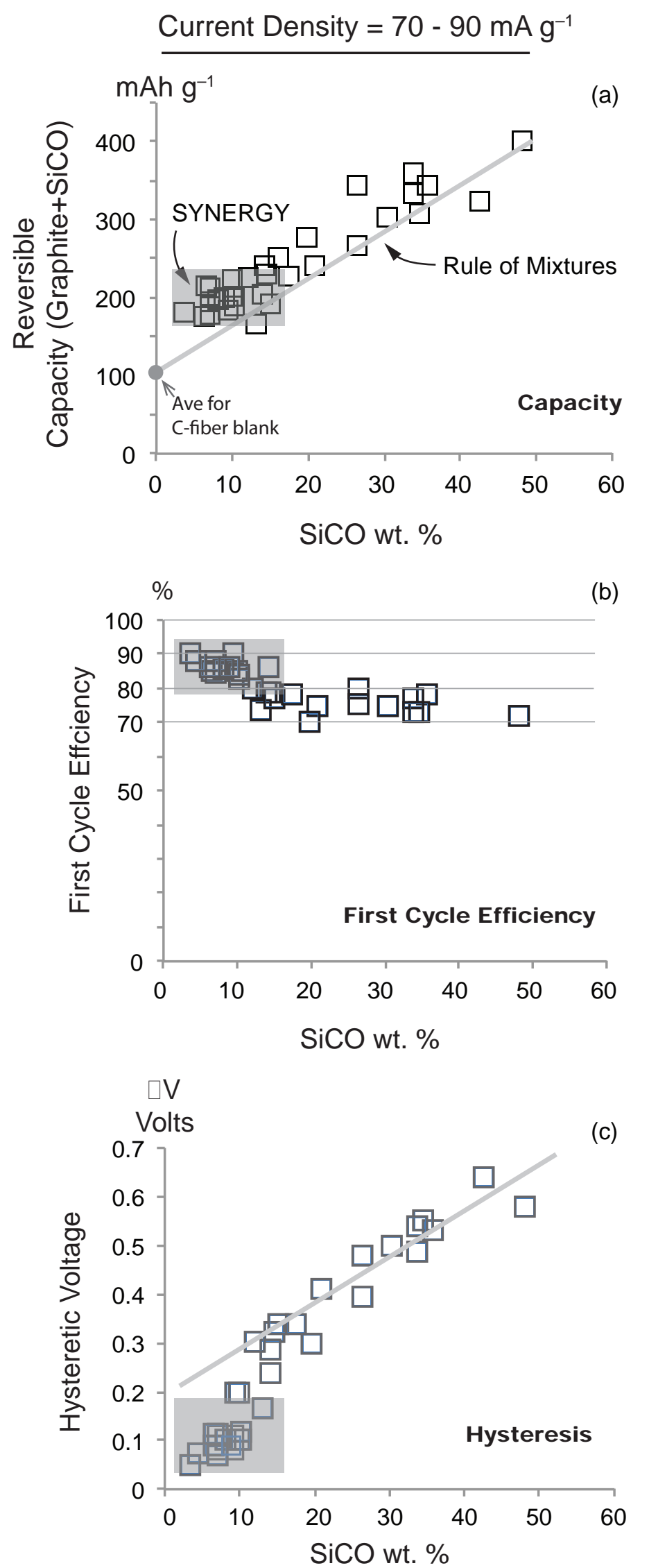

Figure 3. The influence of SiCO wt. \% on (a) the capacity, (b) first cycle efficiency (b), and (c) hysteresis. Significant improvement over SiCO powder is seen for less than $12 \mathrm{wt}$. \%. 

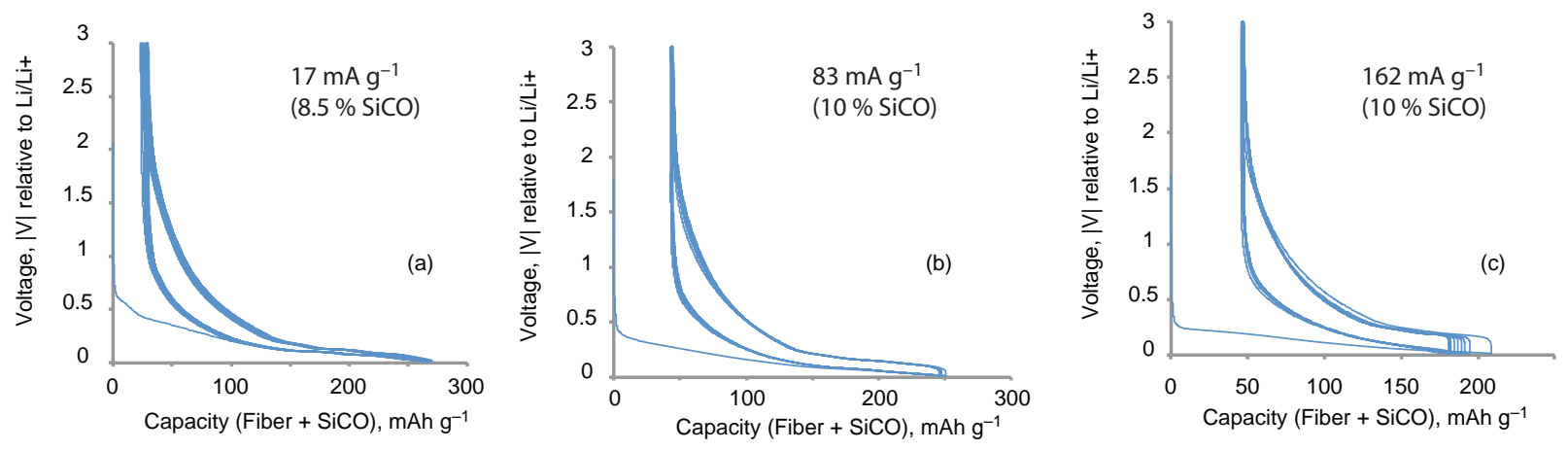

Figure 4. Influence of current density on the cyclic behavior of SiCO ( 10 wt. \%)-GF composite anode. 

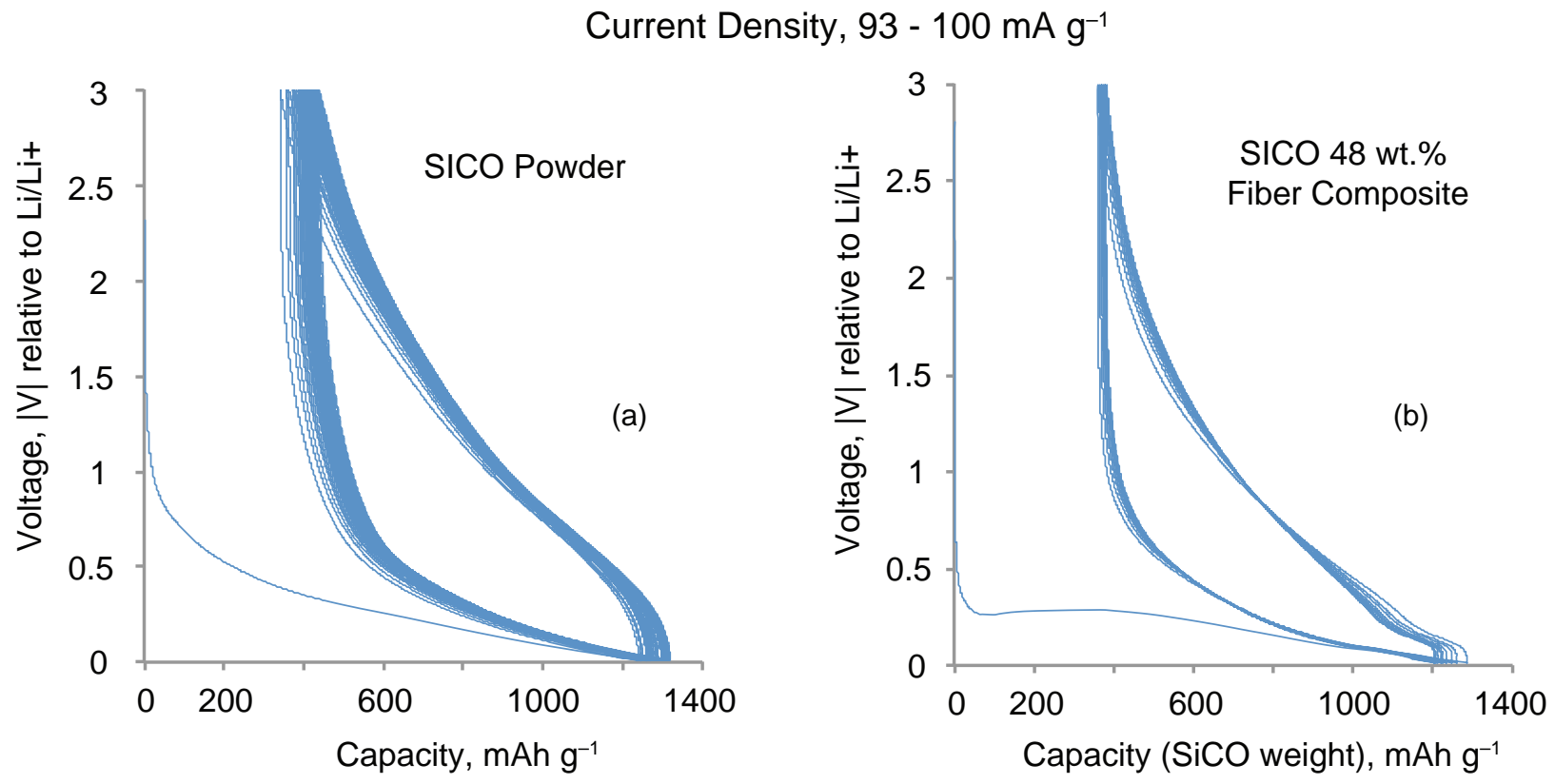

V

Figure 5. (a) The cyclic behavior of SiCO powder anode made by the conventional method. (b) The cyclic behavior of a composite with 48 wt.\% SiCO at the same current density. The capacity has been normalized with respect to the SiCO weight. At this composition the contribution of the graphite fibers to the capacity is minor. 

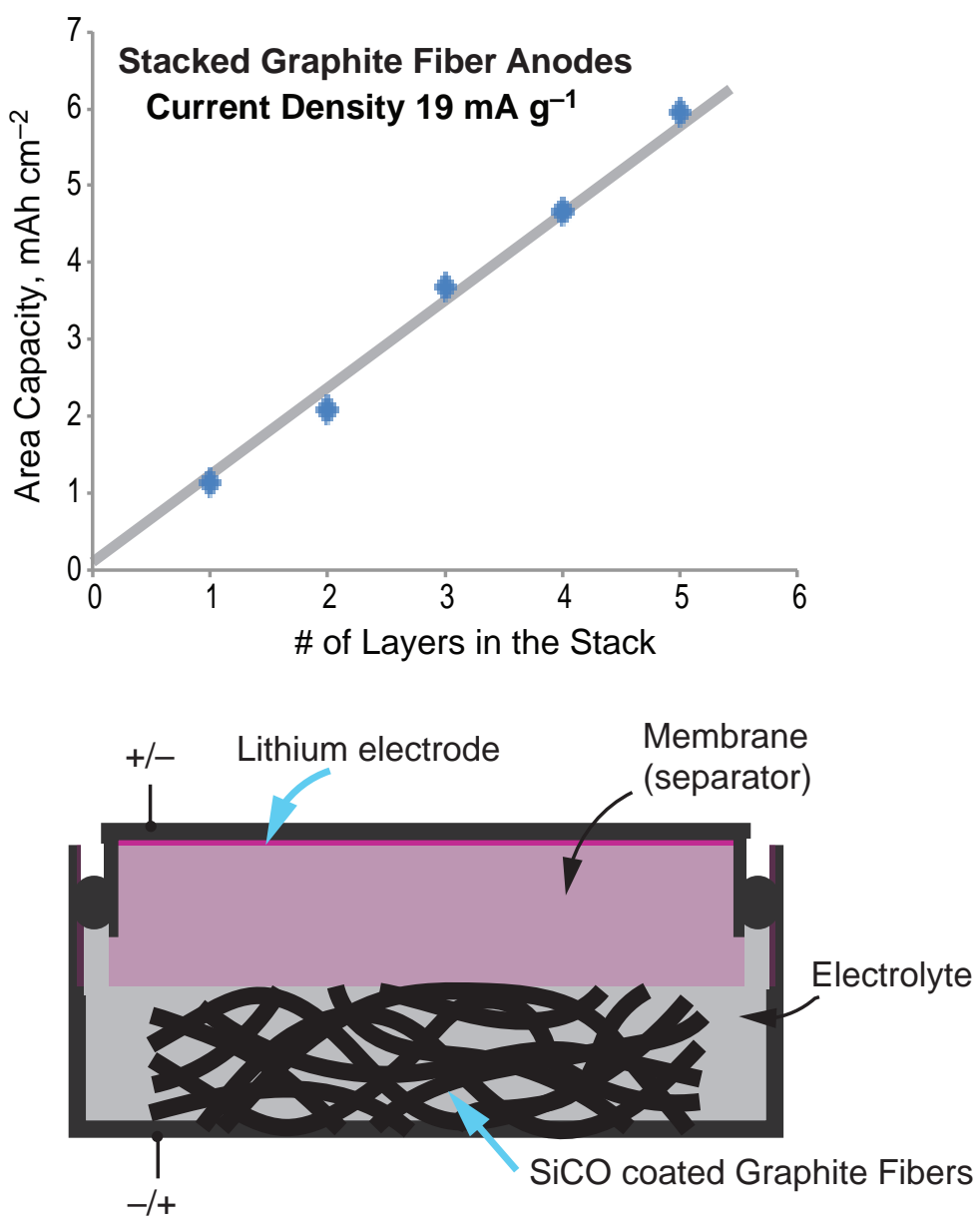

Figure 6. Since all graphite fibers are current collectors, and since the electrolyte makes contact with all of them, the thickness of the anode can be increased without limit to increase the area capacity of the anodes. This thesis is affirmed in these data where the area capacity increases linearly with the number of layers in an anode stack. 

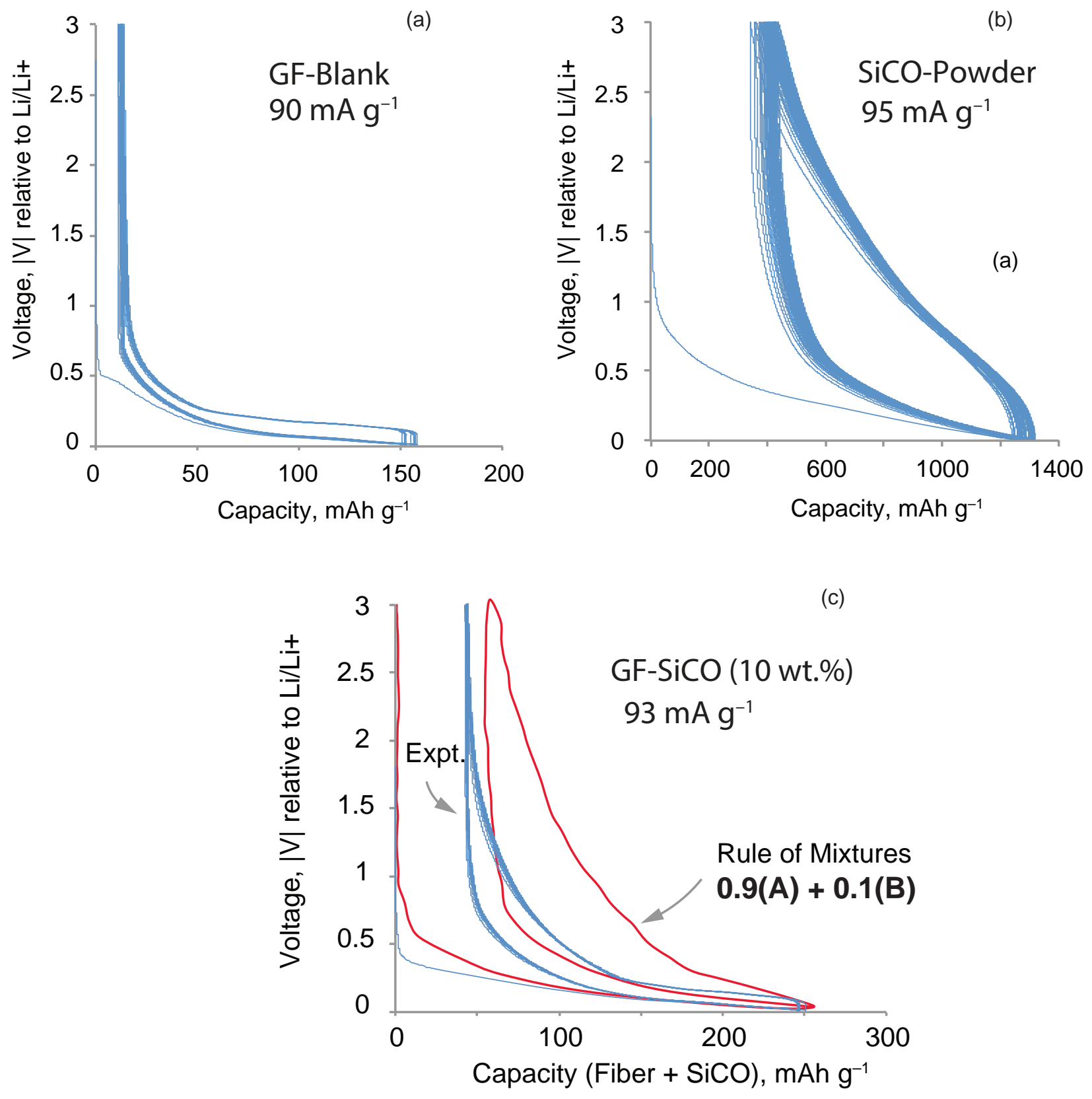

Figure 7. (a) The capacity of GF-Blank and (b) SiCO powder tested separately. (c) The capacity of the composite compared with the calculation from the rule-of-mixtures that combines the results in (a) and (b), using Eq. (3). 


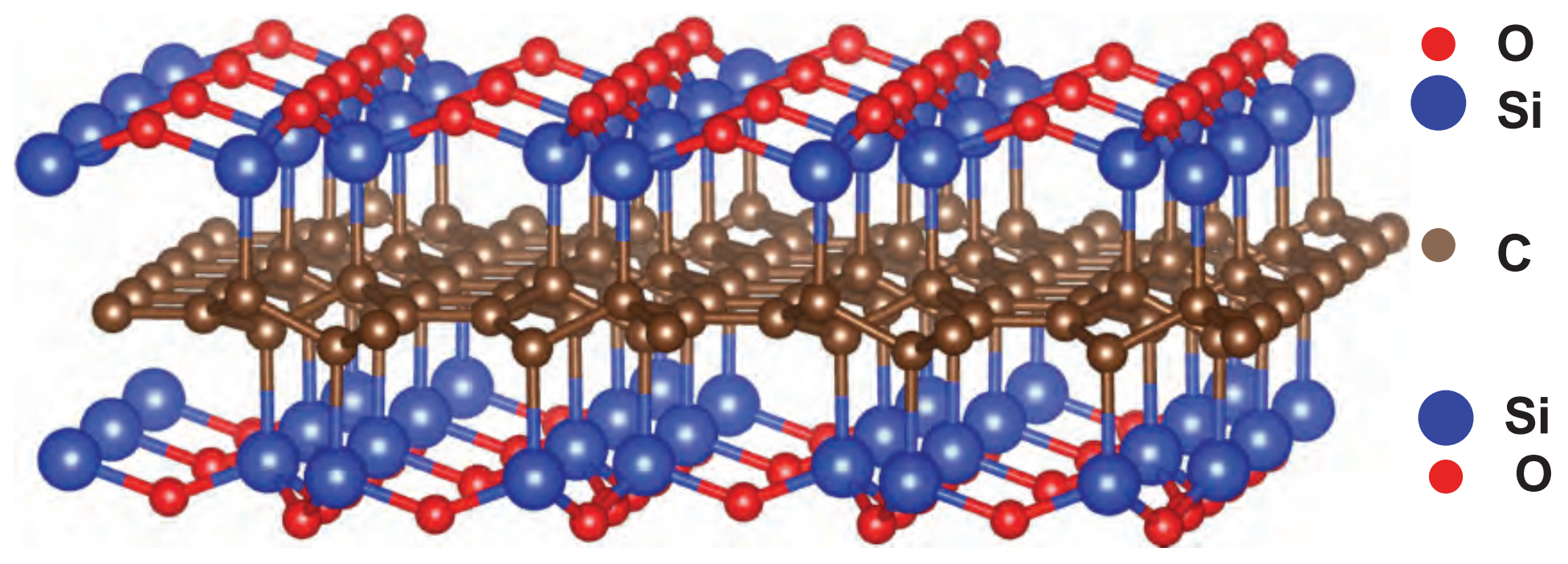

8. A 2-D crystal of monolayers of silicon on graphene with a binding energy of -1.2 eV per silicon atom. The stoichiometry of this structure is $2: 4: 3:: \mathrm{Si}: \mathrm{C}: \mathrm{O}$. 